\title{
Phase-field model for collective cell migration
}

\author{
Sara Najem ${ }^{1}$ and Martin Grant ${ }^{2}$ \\ ${ }^{1}$ Graduate Aerospace Laboratories (GALCIT), California Institute of Technology, Pasadena, California 91125, USA \\ ${ }^{2}$ Physics Department, Rutherford Building, 3600 rue University, McGill University, Montréal, Québec, Canada H3A 2T8
}

(Received 10 November 2015; published 9 May 2016)

\begin{abstract}
We construct a phase-field model for collective cell migration based on a Ginzburg-Landau free-energy formulation. We model adhesion, surface tension, repulsion, coattraction, and polarization, enabling us to follow the cells' morphologies and the effect of their membranes fluctuations on collective motion. We were able to measure the tissue surface tension as a function of the individual cell cortical tension and adhesion and identify a density threshold for cell-sheet formation.
\end{abstract}

DOI: 10.1103/PhysRevE.93.052405

\section{INTRODUCTION}

The evolution of physiological or pathological mechanisms such as wound healing, immune response, embryogenesis, and cancer metastasis is dependent on collective cell migration [1-4]. In the presence of a chemical cue, a single cell chemotaxes and its motion is biased by the extension of the cell's lamellipodia in the direction set by the signal [5]. However, when collective motion is involved, physical forces at the mesoscopic level come to play and the migration is no longer predominantly governed by chemical cues [6-10]. Additionally, in this case the contact with other neighboring cells inhibits the protrusion in the direction of the contact, known as contact inhibition of locomotion (CIL) [3,11]. As well, the motion of the cells [12] is affected by adhesion (mediated by adherens junction proteins $[13,14]$ ), the tension of the cortex (a highly dynamic myosin structure essential to surface tension and to shape control $[15,16])$, coattraction (a mutual chemoattraction mechanism), and collective polarization. Other cell-cell signaling might be triggered, such as regulatory mechanisms by quorum sensing [17].

The interplay of these forces produces the tissue's surface tension. Two competing hypotheses explain this measured effect: the differential adhesion hypothesis (DAH) and the differential interfacial tension hypothesis (DITH) [15]. In models of the DAH, cells are considered as point masses and the tissue surface tension is assumed to be linearly proportional to adhesion molecules' expression levels. This has been experimentally confirmed [18]. However, results from experiments in atomic force microscopy show that the actinmyosin activity affects the cell surface tension, which supports the DITH and the importance of cell shape in determining the tissue surface tension. A more recent experimental study shows that the tissue surface tension is proportional to the ratio of adhesion to cortical tension, where the first increases the cell-cell contact size and the second counteracts it [15].

Density is a key parameter upon which these forces depend and subsequently the tissue surface tension. In various systems, density controls the transition from "order" to "disorder." This is seen in diverse systems ranging from bird flocks to fish schools to colloids and rods [19]. Particularly in high cell density regimes, the separation between cells drops, mutual interaction becomes inevitable, and directional motion can emerge even in the absence of external chemical cues. In this limit only a fraction of "informed cells" is necessary to induce collective migration, and this fraction can be shown to decrease with increasing population size in a wide variety of systems $[12,20]$. For example, a group of promigratory cells could act as a guide to other passive cells through adhesion, which reinforces their action, CIL, and stress-strain realignment by means of physical transfer of information. In addition to that, coattraction, or self-secreted chemical attractant, enhances and maintains the high cell density, acting as a positive feedback on directional migration [21]. Moreover, as the density of the cells increases, the collective migration is known to decelerate at large time and length scales. This is reminiscent of the glass transition in colloidal systems where caging, or the trapping by neighbors, is a signature [22-24]. However, unlike colloidal systems these forces govern the morphological fluctuations the cell undergoes. Thus, understanding the deformability of the cell's membrane becomes essential to understand the transition $[25,26]$.

In simplified models, the motion of the cells is reduced to kinematic systems of coupled differential equations where cells are modeled as point masses and their morphological fluctuations are neglected. Hence, essential aspects of the migration are not recovered. For this purpose we use the phase-field method, which has been used to model individual cell morphology as well as that of a collection of cells [27-30] and derive a system of partial differential equations which follows the evolution of the fields $\phi_{i}$ that depict the cells' morphologies under the effect of chemical cues, local repulsive forces, alignment, adhesion, coattraction, and surface tension in a closed domain. In addition to that, we explore the consequence of the change of the ratio of adhesive forces to cortical tension on tissue surface tension. We compute the mean-square displacement and show that it changes from diffusive to subdiffusive as the density of the cells increases, in addition to investigating the effect of the number of promigratory cells on collective motion.

\section{MODEL}

The dissimilarities between the different phases of a material can be encapsulated in an order parameter, which either can be a conserved quantity, such as the density difference that distinguishes between a material's liquid and gaseous phases, or can vary like the total magnetization that sets apart the paramagnetic and the ferromagnetic phases. In both cases, the order parameter is assigned a distinct value in 


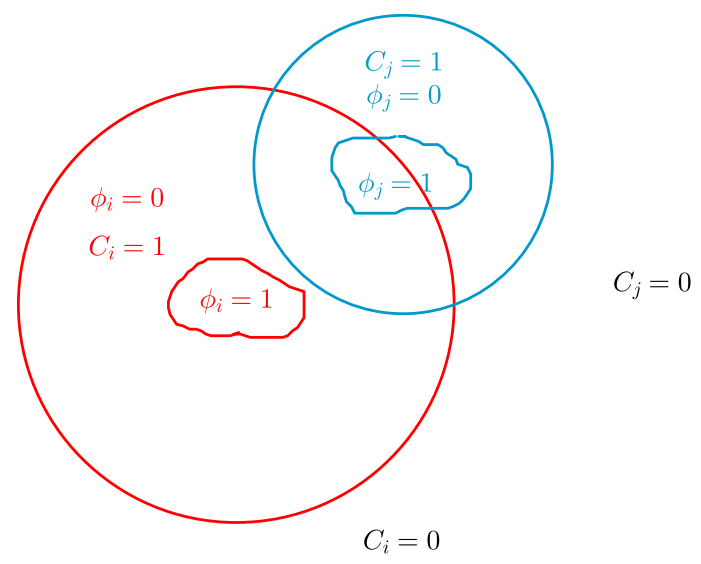

FIG. 1. Fields $\phi_{i}$ and $\phi_{j}$ assigned to cells $i$ and $j$, respectively, together with their interaction ranges $C_{i}$ and $C_{j}$.

each phase and is used to construct the free-energy functional which describes the system's equilibrium properties. The bulk and interfacial energies are given by a power expansion of the order parameter and its spatial derivatives, respectively, which together describe the system's free energy. The coefficients of such expansion are governed by the system's symmetry; for example the coefficients of the odd powers of the derivatives should vanish if the system is invariant under $x \rightarrow-x$. The type of the order parameter determines its driving conjugate forces and thus defines the equation governing the dynamics. These are given, respectively, by Model A and Model B for a varying field and a conserved field [31]. These constitute the Ginzburg-Landau theory which we use to define the order parameter $\phi_{i}$ that takes the value 1 inside the $i$ th cell and 0 outside it [31]. In addition, the field $C_{i}$ sets the range of interaction between the $i$ th cell and its neighbors; it is 1 inside the area of interaction and 0 elsewhere. Figure 1 provides a description of the fields.

The free-energy functional $\mathcal{F}$ which controls the dynamics of $\phi_{i}$ and $C_{i}$ is obtained from the Taylor expansion in powers of the fields and their derivatives, respecting the symmetries of the system. In order to force coexistence of the phases $\phi_{i}=1$ and $\phi_{i}=0$, the values that minimize the bulk-free energy, we truncate the expansion in $\phi_{i}$ to fourth order by defining the double-well energy of the bulk $f\left(\phi_{i}\right)=a \phi_{i}^{2}\left(1-\phi_{i}\right)^{2}$, where $a$ has units of energy per volume. Analogously, we define $f\left(C_{i}\right)=a C_{i}^{2}\left(1-C_{i}\right)^{2}$. The presence of the interface is guaranteed by including $\left(\nabla \phi_{i}\right)^{2}$ and $\left(\nabla C_{i}\right)^{2}$ in the expansion and dropping out odd powers of the derivatives to preserve translational symmetry.

In a continuum mechanics formulation, the adhesion potential given by $W(x)=w e^{-d^{2}(x) / \delta^{2}}$ contributes $-\int_{S} W d s$ to the total energy functional, where $\delta$ determines its interaction range and $d(x)$ measures the distance separating the cells' interfaces. Equivalently, and analogous to the treatment in Ref. [32], in a phase-field description, adhesion is incorporated by adding the term $-\int W_{i}(x) F_{i}\left(\phi_{i}, \nabla \phi_{i}\right) d V$, where in our case $W_{i}=w C_{i} \phi_{i} \sum_{j \neq i}^{N_{c}}\left(1-\phi_{j}\right)$. This term will drive the $i$ th cell to fill the empty space, separating it from its neighboring cells $j$, and effectively lead to cell aggregation. It is approximately $w$ when the cells are separated by empty space within the interaction range $C_{i}$ and 0 otherwise. $F_{i}$ should be 0 away from the diffuse interface of the $i$ th cell and non-negative. We conveniently choose it to be $F_{i}=\phi_{i}\left(1-\phi_{i}\right)$. Additionally, to impose the conservation of the cell volume and its interaction range we add the terms $H_{i} \phi_{i}$ and $H_{2 i} C_{i}$, where $H_{i}$ and $H_{2 i}$ act as external fields coupled to $\phi_{i}$ and $C_{i}$, respectively. The equation of the functional is then given by

$$
\begin{aligned}
\mathcal{F}= & \int \sum_{i=1}^{i=N_{c}}\left[\frac{\epsilon\left(\nabla \phi_{i}\right)^{2}}{2}+f\left(\phi_{i}\right)-w C_{i} \phi_{i}^{2}\left(1-\phi_{i}\right) \sum_{j \neq i}\left(1-\phi_{j}\right)\right. \\
& \left.+H_{i} \phi_{i}+\frac{\epsilon\left(\nabla C_{i}\right)^{2}}{2}+f\left(C_{i}\right)+H_{2 i} C_{i}\right] d V
\end{aligned}
$$

\section{EQUATIONS}

The equations for the evolution of $\phi_{i}$ and $C_{i}$ conserved [31]. Explicitly, these are given by $\frac{d \phi_{i}}{d t}=\frac{\partial \phi_{i}}{\partial t}+\vec{v}_{i} \cdot \nabla \phi_{i}=$ $\Gamma \nabla^{2}\left[\frac{\delta \mathcal{F}}{\delta \phi_{i}}\right]$ and $\frac{d C_{i}}{d t}=\frac{\partial C_{i}}{\partial t}+\vec{v}_{i} \cdot \nabla C_{i}=\Gamma \nabla^{2}\left[\frac{\delta \mathcal{F}}{\delta C_{i}}\right]$, where $\Gamma$ is the fields' mobility and $\vec{V}_{i}$ is the cell's velocity. The governing equations are therefore given by

$$
\begin{aligned}
\frac{\partial \phi_{i}}{\partial t}= & -\Gamma \nabla^{2}\left[\epsilon \nabla^{2} \phi_{i}-f_{\phi_{i}}+w C_{i}\left(2 \phi_{i}-3 \phi_{i}^{2}\right) \sum_{j \neq i}\left(1-\phi_{j}\right)\right] \\
& -\vec{\nabla} \cdot\left(\vec{V}_{i} \phi_{i}\right), \\
\frac{\partial C_{i}}{\partial t}= & -\Gamma \nabla^{2}\left[\epsilon \nabla^{2} C_{i}-f_{C_{i}}\right]-\vec{\nabla} \cdot\left(\vec{V}_{i} C_{i}\right),
\end{aligned}
$$

where $\epsilon$ is proportional to the surface tension, $\Gamma \nabla^{2} H_{i}=-\phi_{i} \vec{\nabla} \cdot \vec{V}_{i}, \Gamma \nabla^{2} H_{2 i}=-C_{i} \vec{\nabla} \cdot \vec{V}_{i}$, and $f_{\phi_{i}}$ and $f_{C_{i}}$ are the derivatives of $f\left(\phi_{i}\right)$ and $f\left(C_{i}\right)$ with respect to $\phi_{i}$ and $C_{i}$, respectively.

We define the velocity field to be

$$
\vec{V}_{i}=\left(\vec{v}_{i, c c}+\sum_{j \neq i}^{N_{c}} \vec{v}_{j, s s}+\vec{u}_{i}\right) \sum_{j \neq i}^{N_{c}}\left(1-\phi_{j}\right)-r \sum_{j \neq i}^{N_{c}} \vec{\nabla} \phi_{j} .
$$

The first term includes the chemotactic effect $\vec{v}_{i, c c}=\vec{\nabla} c$ where $c$ is a Gaussian centered at a fixed point in the domain.
Including the term $\sum_{j \neq i}^{N_{c}}\left(1-\phi_{j}\right)$ in the definition of the velocity forces the cells to move in empty space and to avoid 


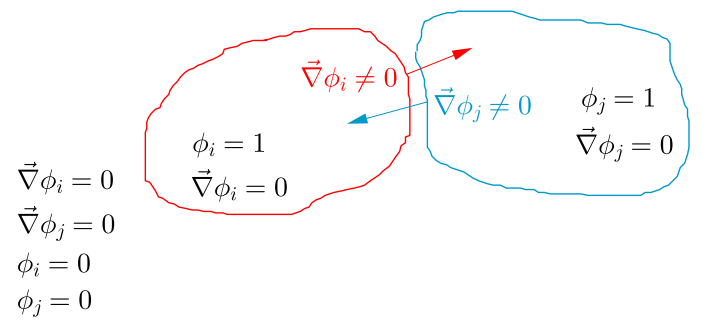

FIG. 2. The scheme shows the repulsive forces exerted by the cells' membranes on each other.

overlap with others: it is 0 at the cells' locations, which is equivalent to the CIL effect. The second term, $\sum_{j \neq i}^{N_{c}} \vec{v}_{j, s s}$, gives guiding by self-secreted chemical cues, which together with the first define the polarization contribution. The third term includes self-propulsion and alignment; it also differentiates between leading and following cells. More precisely,

$$
\vec{u}_{i}=\vec{v}_{i} \sum_{j \neq i}^{N_{c}}\left(1-C_{j}\right)+C_{i}\left(1-H_{\left|\vec{v}_{i, c c}\right|}\right) \frac{\sum_{j \neq i}^{N_{c}} C_{j} \vec{v}_{j}}{\left(s+\sum_{j \neq i}^{N_{c}} C_{i} C_{j}\right)},
$$

where the term $\vec{v}_{i}$ is the self-propulsive term, as in [30,33], $s \ll 1$ is smoothing parameter, and $H$ is the heaviside step function. The distinction between leading and following cells, or those which can detect an external chemical gradient and those which cannot, respectively, is captured by multiplying the alignment term by $\left(1-H_{\mid \vec{v}_{i, c c}}\right)$, which is 0 when the cell is a leader; it moves unaffected by the motion of the others. More precisely, the alignment term, the last ratio of Eq. (4), realigns the velocity field of a follower cell located within the interaction range of its neighbors $C_{j}$ to their average velocity, while the first term in Eq. (4) keeps the cell unaffected if it is outside all ranges $\sum_{j \neq i}^{N_{c}} 1-C_{j}$ as shown in Fig. 1. Additionally, to incorporate coattraction, we define the velocity due to a self-secreted chemical cue to be proportional to the gradient of a Gaussian $G_{i}$, centered at the $i$ th cell's position: $\vec{v}_{i, s s}=\vec{\nabla} G_{i}$. Finally, the last term in Eq. (3) guarantees repulsion between cells as they detect each other's membranes, as seen in Fig. 2.

\section{RESULTS}

Equation (2) was solved using a finite element algorithm and the conditions for numerical stability and critical cell size were met [31,34], with periodic boundary conditions imposed on the computational domain. First, we note that there are two time scales in the dynamics: one for the cells to relax to their equilibrium shapes and the other is related to translational motion. The first is 1 order of magnitude faster than the second and thus our choice of parameters had to satisfy this condition as well [30]. Also, the dynamics of $G_{i}$ were solved using a fictitious time integration method, since we assume that selfsecreted chemical gradients' dynamics are fast on the time scale of any other mechanism. Figure 3 is a sample result.

We explored the effect of density on the net polarization $P$ given by Eq. (5), in the limit where $\vec{v}_{i, c c}=0$, such that is no external chemical cue is present nor self-secreted chemical

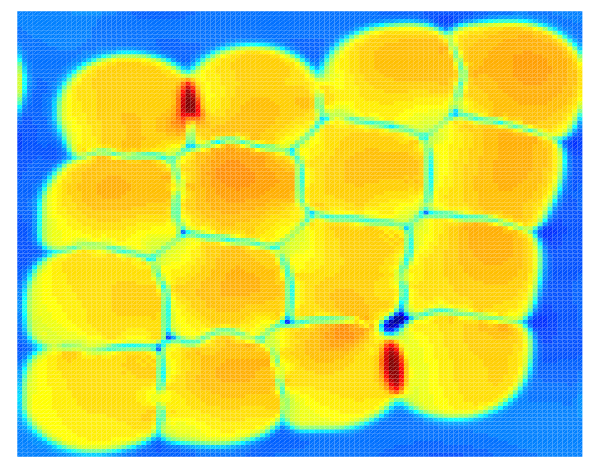

FIG. 3. The figure shows an aggregate of $N_{c}=16$ cells in yellow for $\frac{w}{\sigma}=0.5, b=0, r=30, \Gamma=1, a=16$, and cell radii $R_{c}=4$.

cues $\vec{v}_{i, s s}=0$ :

$$
P=\left\langle\frac{1}{N_{c}}\left|\sum_{i}^{N_{c}} \frac{\vec{x}_{i}\left(t+h_{t}\right)-\vec{x}_{i}(t)}{\left|\vec{x}_{i}\left(t+h_{t}\right)-\vec{x}_{i}(t)\right|}\right|\right\rangle_{t},
$$

where $\vec{x}_{i}$ is the position of the center of mass of the $i$ th cell and $h_{t}$ is the time step size of the simulation. $P$ measures the movement polarization. We varied the nondimensionalized density and measured its effect on $P$ as shown in Fig. 4. The dependence is given by $P \propto\left(n-n_{0}\right)^{\gamma}$, where $\gamma=$ $0.53 \pm 0.05$ and $n_{0}=0.16 \pm 0.02$, which is analogous to the behavior at a mean-field second-order phase transition. For each density, we varied the ratio of adhesive $w$ to cortical forces $\sigma=\frac{4}{3} \sqrt{\frac{a}{(2 \epsilon)}}$ for values ranging between 0.1 and 0.5 . These are represented by the lower and the upper limits of the bars, respectively. It is worthwhile noting that the restriction on the range of values $w / \sigma$ can take is imposed by the stability of the algorithm. Subsequent to the increase in the ratio $w$ to $\sigma$, the polarized movement increases, which is depicted by the

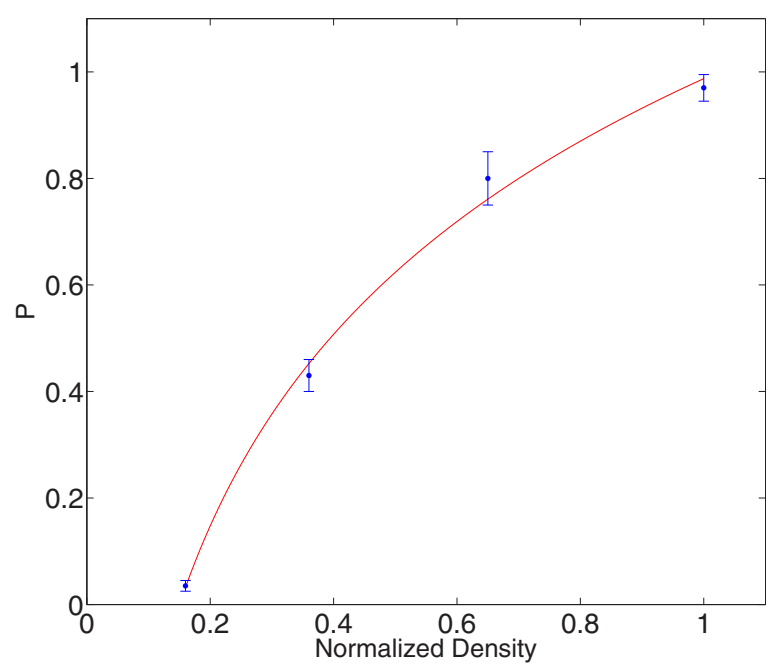

FIG. 4. The figure shows the polarization as a function of density. When $w / \sigma$ is increased, for each of the densities, the corresponding $P$ increases from the lower limit of the bar to its upper limit. The curve is given by $P=\left(n-n_{0}\right)^{\gamma}$, where $\gamma=0.53 \pm 0.05$ and $n_{0}=0.16 \pm 0.02$. 


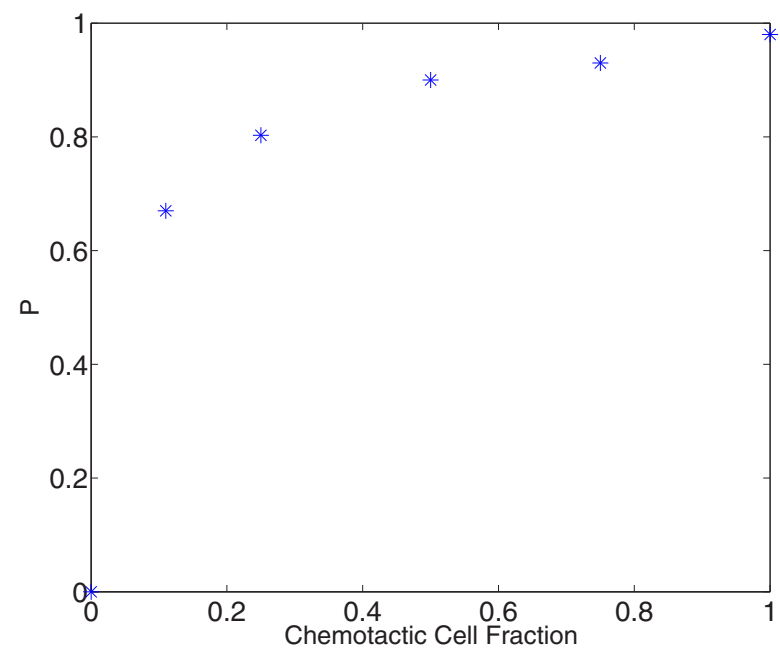

FIG. 5. The figure shows the polarization as a function the fraction of chemotactic cells.

upper bound of the bars. When cell-cell adhesion is increased, cells are more likely going to stick together and the alignment term in our equation will contribute in an increase in the net polarization. All this confirmed that a net polarization would spontaneously occur in the absence of any external cue as a result of the combined effects of alignment and adhesion [21]. Throughout we fixed the range or the radius of $C_{i}$ to 1.3 larger than the size of the cell $\phi_{i}$, below which no net polarization is observed.

On the other hand, in low-density regimes and when polarization could not result from the interplay of the abovementioned forces, external chemical cues are known to be essential for collective migration to occur. However, only a fraction of the population is needed to be promigratory to induce polarization [21]. Hence, we assign nonzero $\vec{v}_{i, c c}$ for a varying fraction of cells and measure the net polarization in each case. We recover the results in [21] as shown in Fig. 5.

Additionally, the effect of density change on the meansquared displacement (MSD) is measured. Cells exhibit rapid diffusion for moderate densities $\propto t$; the mechanism slows down dramatically as the density increases. In Fig. 6, we plot our data of the MSD for increasing cell densities and follow its change from linear to nonlinear. In addition, we follow the intermediate structure factor which, for the high-density regime, is characterized by the two relaxations times $\beta$ and $\alpha$. The first time scale is fast and corresponds to diffusion in a confined "cage," analogous to the phenomenology associated with a structural glass transition. The second slower scale is the time necessary to diffuse out of the cage. This interpretation explains the change in the mean-squared displacement and suggests that the self-propelled cells undergo a dynamic transition, analogous to a glass transition [35].

For high densities a cell sheet will form, which is characterized by a tissue surface tension $T_{\mathrm{st}}$. In order to measure its dependence on the ratio of adhesion to the individual cell cortical tension, we solve Eq. (2) with fixed initial conditions while varying the ratio, as shown in Fig. 7. The data are

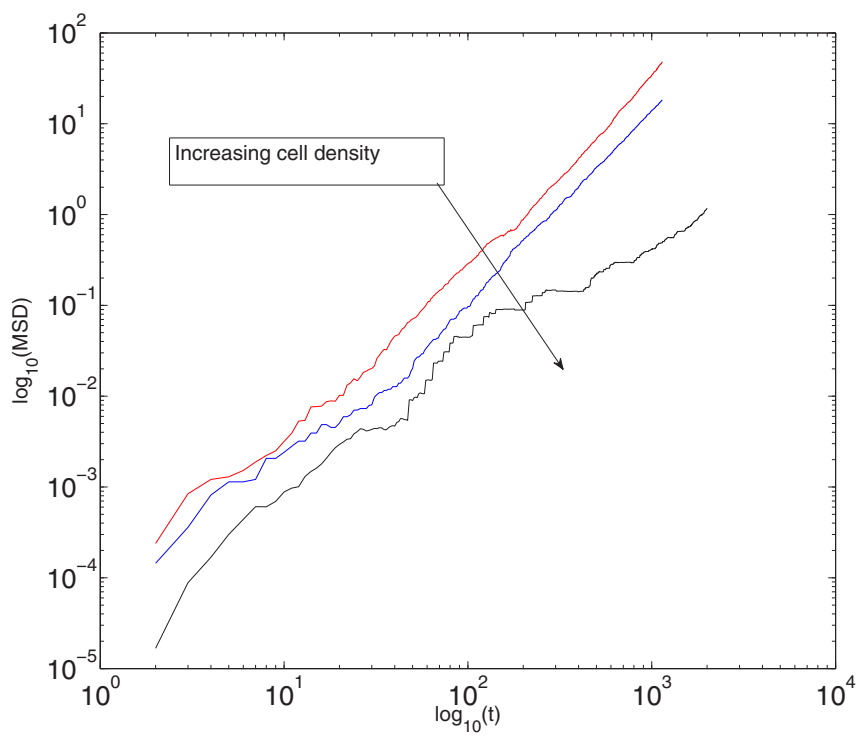

FIG. 6. The figure shows MSD as function of time for densities $n=0.16, n=0.36$, and $n=0.65$, respectively, in red (top line), blue (middle line), and black (bottom line) on a log - log scale. The low-density results have asymptotic slopes consistent with diffusion.

compared to the line $T_{\mathrm{st}} / \sigma=2 \mathrm{w} / \sigma$, which is in accordance with the computations reported in [15]. Analogous to fluids, the tissue surface tension is $T_{\mathrm{st}}$, which measures the response of the tissue to variation in surface area, and is proportional to the energy difference between the inner cells and those at the surface $T_{\text {st }}=\left(W_{\text {surface cell }}-W_{\text {bulk cell }}\right) / L$, where $L$ is the projected length of a single cell onto the surface of the aggregate and the energy of the cell is given by $W_{\text {cell }}=$ $\left(\sigma-\frac{w}{2}\right) P_{c}+\sigma P_{\mathrm{NC}}$, where $P_{c}$ and $P_{\mathrm{NC}}$ are, respectively, the contact perimeter of the cell with others and its noncontact perimeter [26].

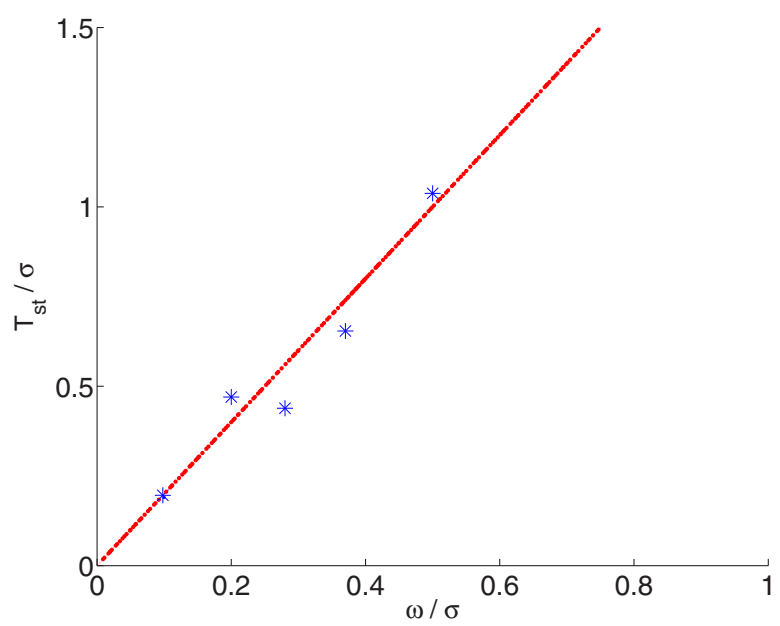

FIG. 7. We vary the tissue surface tension for $n=0.65$ with fixed initial conditions. The figure shows the dependence of the tissue surface tension on the ratio of adhesion to cortical tension. The red line is $T_{\text {st }} / \sigma=2 w / \sigma$, which equivalent to the differential adhesion hypothesis in two spatial dimensions. 


\section{CONCLUSION}

In this article, we have used the phase-field method to model collective cell migration and presented a mechanistic approach which included all the forces that are known to govern cell-cell interaction as well as cell membrane morphology. We measured the transition to order as a function of cell density and recovered the leadership behavior of promigratory cells. Incorporating these forces, we were able to model cell morphologies and membrane fluctuations and consequently predict a dependence of tissue surface tension on adhesion and cortical tension.

\section{ACKNOWLEDGMENTS}

We acknowledge support from the Natural Sciences and Engineering Research Council of Canada, le Fonds de recherche du Québec-Nature et technologies, and discussions with Iain Couzin and members of the Couzin Lab at Princeton University.
[1] S. Battersby, Proc. Natl. Acad. Sci. USA 112, 7883 (2015).

[2] P. Friedl and D. Gilmour, Nat. Rev. Mol. Cell Biol. 10, 445 (2009).

[3] A. J. Kabla, J. R. Soc. Interface 9, 3268 (2012).

[4] M. Basan, J. Elgeti, E. Hannezo, W.-J. Rappel, and H. Levine, Proc. Natl. Acad. Sci. USA 110, 2452 (2013).

[5] P. K. Mattila and P. Lappalainen, Nat. Rev. Mol. Cell Biol. 9, E182 (2005).

[6] N. Gov, Nat. Mater. 10, 412 (2011).

[7] P. Dieterich, R. Klages, R. Preuss, and A. Schwab, Proc. Natl. Acad. Sci. USA 105, 459 (2008).

[8] J. Löber, F. Ziebert, and I. S. Aranson, Nat. Sci. Rep. 5, 9172 (2015).

[9] T. E. Angelini, E. Hannezo, X. Trepat, J. J. Fredberg, and D. A. Weitz, Phys. Rev. Lett. 104, 168104 (2010).

[10] D. T. Tambe et al., Nat. Mater. 10, 469 (2010).

[11] S. R. Vedula, A. Ravasio, C. T. Lim, and B. Ladoux, Physiology 28, 370 (2008).

[12] L. Coburn, L. Cerone, C. Torney, I. Couzin, and Z. Neufeld, Phys. Biol. 10, 046002 (2013).

[13] U. Schwarz and S. Safran, Rev. Mod. Phys. 85, 1327 (2013).

[14] V. Braga, Nat. Cell Biol. 2, E182 (2000).

[15] M. L. Manning, R. A. Foty, M. S. Steinberg, and E. M. Schoetz, Proc. Natl. Acad. Sci. USA 107, 12517 (2010).

[16] G. Salbreux, G. Charras, and E. Paluch, Trends Cell Biol. 22, 536 (2012).

[17] M. B. Miller and B. L. Bassler, Annu. Rev. Microbiol. 55, 165 (2001).

[18] R. A. Foty and M. S. Steinberg, Dev. Biol. 278, 255 (2005).
[19] M. C. Marchetti et al., Rev. Mod. Phys. 85, 1143 (2013).

[20] B. Szabó, G. J. Szöllösi, B. Gönci, Z. Jurányi, D. Selmeczi, and T. Vicsek, Phys. Rev. E 74, 061908 (2006).

[21] M. L. Woods, C. Carmona-Fontaine, C. P. Barnes, I. Couzin, R. Mayor, and K. M. Page, PLoS One 9, e104969 (2014).

[22] P. M. Reis, R. A. Ingale, and M. D. Shattuck, Phys. Rev. Lett. 98, 188301 (2007).

[23] D. Bi, J. H. Lopez, and J. M. Schwartz, Soft Matter 10, 1885 (2014).

[24] J. Sun, D. J. Earl, and M. W. Deem, Phys. Rev. Lett. 95, 148104 (2005).

[25] T. S. Deisboeck and I. Couzin, Bioessays 31, 190 (2009).

[26] T. Angelini, E. Hannezo, X. Trepat, M. Marquez, J. J. Fredberg, and D. A. Weitz, Proc. Natl. Acad. Sci. USA 108, 4714 (2011).

[27] G. R. Lázaro, A. Hernández-Machado, and I. Pagonabarrag, Soft Matter 10, 7195 (2014).

[28] G. R. Lázaro, A. Hernández-Machado, and I. Pagonabarrag, Soft Matter 10, 7207 (2014).

[29] J. Löber, F. Ziebert, and I. S. Aranson, Soft Matter 10, 1365 (2014).

[30] B. Palmieri, Y. Bresler, D. Wirtz, and M. Grant, Nat. Sci. Rep. 5, 11745 (2015).

[31] N. Provatas and K. Elder, Phase Field Methods in Materials Science and Engineering (Wiley-VCH, New York, 2010).

[32] J. Zhang, S. Das, and Q. Du, J. Comput. Phys. 228, 7837 (2008).

[33] E. A. Codling, M. J. Plank, and S. Benhamou, J. R. Soc. Interface 5, 813 (2008).

[34] P. Yue, C. Zhou, and J. J. Feng, J. Comput. Phys. 223, 1 (2006).

[35] L. Berthier, Phys. Rev. Lett. 112, 220602 (2014). 